Implications for Guideline Developers/Users RE-AIM framework is a useful tool to evaluate the effect of using guidelines and develop strategies to disseminate them.

\section{P282 MANAGEMENT OF PATIENTS WITH BIPOLAR DISORDER: AN ADAPTED CLINICAL PRACTICE GUIDELINE FROM KING SAUD UNIVERSITY, KING KHALID UNIVERSITY HOSPITAL, CLINICAL PRACTICE GUIDELINES COMMITTEE, FACULTY OF MEDICINE, DEPARTMENT OF PSYCHIATRY}

1,2,4E Abahussain, ${ }^{2,4,5}$ S Fatani, , 1,2,4 M Altuwarigi, ${ }^{2,4,6} \mathrm{~L}$ Al-Ansary, ${ }^{2,3,4} \mathrm{Y}$ Amer. ${ }^{1}$ Psychiatry Department, College of Medicine, King Saud University, Riyadh, Saudi Arabia; ${ }^{2}$ CPGs Committee and subcommittee, KSUHs, Riyadh, Saudi Arabia; ${ }^{3}$ Quality Management Department, KSUHs, Riyadh, Saudi Arabia; ${ }^{4}$ King Khalid University Hospital, Riyadh, Saudi Arabia; ${ }^{5}$ Pharmacy Department, RiyadhSaudi Arabia Shaikh Bahamdan Chair for Evidence-Based Healthcare \& Knowledge Translation, Riyadh Saudi Arabia

\section{0:1136/bmjqs-2013-002293.238}

Objectives Adaptation of CPGs for Treatment of Bipolar disorder in King Khalid University Hospital, Psychiatry Department Methods The ADAPTE process for CPGs adaptation. Results: the final decision of the panel after full assessments of 3 source CPGs was full acceptance (adoption) of the Canadian Network for Mood and Anxiety Treatments (CANMAT) and International Society for Bipolar Disorders (ISBD) collaborative update of CANMAT guidelines for the management of patients with bipolar disorder (updated 2009).

Results Examples of Recommendations Lithium, valproate, and several atypical antipsychotics monotherapy is recommended to be used as first line treatments for acute mania, combination pharmacology with antipsychotics and mood stabiliser can be used as first line option. Paliperidone monotherapy and asenapine alone or in combination with lithium or divalproex can be used as a second line treatment; tamoxifen is suggested as a third line augmentation option. For the Management of bipolar depression, lithium, lamotrigine, and quetiapine monotherapy, olanzapine plus selective serotonin reuptake inhibitor (SSRI), and lithium or divalproex plus SSRI bupropion are first-line options. Adjunctive modafinil is recommended as a second-line option. lithium, lamotrigine, valoproate and olanzapine are firstline options for maintenance treatment of bipolar disorder. Conclusion New data support the use of quetiapine monotherapy and adjunctive therapy for the prevention of manic and depressive events, aripiprazole monotherapy for the prevention of manic events, and risperidone long-acting injection monotherapy and adjunctive therapy, and adjunctive ziprasidone for the prevention of mood events.

\section{P283 DEVELOPMENT OF A CLINICAL PRACTICE GUIDELINE ON INTRAVENOUS THERAPY USING GRADE: INTEGRATING AVAILABLE EVIDENCE AND EXPERT OPINION}

${ }^{1} E$ Briones, ${ }^{2} I$ Marín-León, ${ }^{3} \mathrm{M}$ Buzón, ${ }^{4} \mathrm{R}$ García-Aguilar, ${ }^{5} \mathrm{~A}$ Romero-Tabares, A, RomeroAlonso ${ }^{6}$, J. Medrano ${ }^{7}$, E. Calderón ${ }^{8}$, L. López, ${ }^{9}$ ' Public Health Unit. SAS, Sevilla, Spain; ${ }^{2}$ Virgen del Rocio Hospital. SAS, Sevilla, Spain; ${ }^{3}$ FISEVI.Virgen del Rocio Hospital. SAS, Sevilla, Spain; ${ }^{4}$ Agencia de Evaluación de Tecnologías Sanitarias (AETSA), Sevilla, Spain; ${ }^{5}$ Agencia de Evaluación de Tecnologías Sanitarias (AETSA), Sevilla, Spain; ${ }^{6}$ Valme Hospital. SAS Sevilla, Spain; 'Virgen del Rocio Hospital. SAS Sevilla Spain Virgen del Rocio Hospital. SAS Sevilla Spain Virgen del Rocio Hospital. SAS Sevilla Spain

10:1136/bmjqs-2013-002293.239
Background Intravenous therapy is one of the most frequently used interventions in health care, with increasing complexity and duration of treatments. A clinical practice guideline (CPG) on this topic was developed within the framework of the Spanish programme (National Health System) aimed to provide recommendations on intravenous therapy best practice.

Objective To describe CPG development process, considering methodological issues, such as updating and adapting existing guidelines and expert inputs.

Methods Using the GRADE approach, an evidence synthesis was developed for each clinical question, including adoption or update of valid recommendations from selected guidelines. Expert panel followed methodology proposed by Jaescke et al with predefined consensus criteria to categorise recommendations.

Results After assessment with AGREE II instrument, 3 guidelines were considered highly recommended and selected for adaptation/updating. Evidence synthesis and recommendations for each 63 initial clinical question were drafted and sent to the expert panel who voted individually. Areas of disagreement were identified and discussed in a face-to-face meeting. After the second voting round 55 recommendations remained, of which 27 were rated as strong, 12 as weak and 14 as good practice recommendations. Six of them were adopted/updated from previous guidelines. In 2 cases there was no agreement in the panel and no recommendation was issued.

Discussion GRADE proved to be a useful method to develop a complex guideline, incorporating evidence from previous guidelines and expert opinion. The method was well accepted and followed by the panel and improved the elaboration of recommendations.

\section{P284 A SURVEY OF THE UPDATING OF CLINICAL PRACTICE GUIDELINES IN CHINA}

${ }^{1,2} \mathrm{Y}$ Chen, ${ }^{1,2} \mathrm{X}$ Wang, ${ }^{1,2} \mathrm{Q}$ Wu, ${ }^{1,2} \mathrm{Q}$ Wang, ${ }^{1,2} \mathrm{D}$ Wei, ${ }^{1,2} \mathrm{~L}$ Yao, ${ }^{1,2} \mathrm{~F}$ Liang, ${ }^{3} \mathrm{M}$ Wang,

$1,2 \mathrm{~K}$ Yang. 'Evidence-Based Medicine Center of Lanzhou University, Lanzhou, China; ${ }^{2}$ Chinese GRADE Center, Lanzhou, China; ${ }^{3}$ The First Hospital of Lanzhou University, Lanzhou, China

\section{0:1136/bmjqs-2013-002293.240}

Background Clinical Practice Guidelines (CPGs) should be updated when new evidence suggests the need for modification of clinically important recommendations. Some guideline handbooks suggest that CPGs should update every 2 to 5 years. Little is known about the updating of CPGs in China.

Objectives To investigate the current situation of the updating of CPGs in China.

Methods We searched WangFang, VIP, Chinese National Knowledge Infrastructure (CNKI) and Chinese Biomedical Literature Database (CBM) up to December 2012. And then we screened all included papers.

Results A total of 380 Chinese CPGs were included. There were 345 of guidelines (91\%) had not been updated. 35 (9\%) were updated and the average update cycle of them was 5.6 years (from 1 to 9 years). No guideline described the details of methods of uptodate .

Conclusions Updating the CPGs is important for the better dissemination and utilisation. But in China, most of the CPGs had never been updated, and the average update cycle (5.6 years) of the updated is longer than international standard (5 years). We suggest Chinese guideline developers should pay more attention to the updating the guideline in time. 